\title{
PLANETARY ACCRETION DEBRIS AND IR EXCESSES IN OPEN STELLAR CLUSTERS
}

\author{
D. E. Backman, J. R. Stauffer, and F. C. Witteborn \\ NASA Ames Research Center \\ Moffett Field, CA 94035-1000
}

\section{Poster Paper}

We have initiated an infrared survey of $550 \mathrm{~A}-\mathrm{G}$ main sequence stars in clusters with ages less than 1 billion years. This survey consists primarily of coadded IRAS survey photometry. The goals are: (1) to identify infrared excesses which might be attributable to protoplanetary accretion/collisional debris, and (2) to search for a possible trend with stellar age. 